\title{
Parametric response map (PRM) is a promising tool for the monitoring of post traumatic cerebral oedema
}

\author{
J Grèze ${ }^{1,2^{*}}$, B Lemasson², E Barbier ${ }^{2}$, J-F Payen ${ }^{1,2}$, P Bouzat ${ }^{1,2}$ \\ From ESICM LIVES 2015 \\ Berlin, Germany. 3-7 October 2015
}

\section{Introduction}

Cerebral edema (CE) is the key factor for the development of secondary brain injuries after severe traumatic brain injury (TBI). This edema may be assessed using the averaged Apparent diffusion coefficient (ADC) measured by diffusion-weighted MRI in specified ROI. However, this approach does not take into account the complexity and the heterogeneity of the post traumatic edema. The parametric response map (PRM), a voxel-based analysis technique, is a promising tool to better investigate spatially dispersed changes of ADC over time (1).

\section{Objectives}

In this study, we compared the PRM analysis to the classical ADCaveraged approach to characterize evolution of the ADC in the hours following a rat TBI.

\section{Methods}

Eighteen male Wistar rats were traumatized (TBI group) and 7 sham operated rats were used for the control group. Injury was induced using the impact-acceleration protocol (2). Diffusion-weighted images were acquired using an echo-planar, spin-echo, sequence. Acquisitions were performed before the trauma (H.ref), immediately after (H0), 60 min after (H1) and $120 \mathrm{~min}(\mathrm{H} 2)$ after the trauma. Two ROI were contoured, one including the cortex (Cortical ROI) and the other including most of the brain (Brain ROI). For each rat, each time point and ROI, PRM was used to analyze, voxel-wise, changes in ADC. PRM was performed in specified ROI by calculating the difference in ADC values of each voxel between H.ref and the values of the other time points. A variation threshold $\left(100 \mu \mathrm{m}^{2} / \mathrm{s}\right)$ was defined as the variation value below which $95 \%$ of the voxels in the sham rats were considered as stable by the PRM analysis. We computed then the fraction of voxels whose ADC significantly increased (PRM+: red), the fraction of voxels whose ADC significantly decreased (PRM-: blue), and the fraction of voxels whose $\mathrm{ADC}$ was unchanged (PRM0: green). In addition, mean ADC inside the ROI was computed.

\section{Results}

Classical approach showed a difference between TBI group and Sham group in Cortical ROI $(\mathrm{p}=0.04)$ but not in Brain ROI ( $p=0.32)$. PRM approach on the Brain ROI detected edematous processes: TBI group presented statistically fewer PRM0 voxels than Sham group at every time point $(\mathrm{p}<0.05)$, with a dual increase of red and blue voxels. We sorted traumatized rats based on their mean ADC at $\mathrm{H} 2$ in vasogenic (increased ADC) or cytotoxic (decreased ADC) subgroups, and PRM analysis performed immediately after TBI was able to precisely predict the type of edema at $\mathrm{H} 2$ (specificity $100 \%$, sensibility $88.7 \%$ ).

\section{Conclusions}

PRM analysis is a valuable tool for analyzing the complexity of post traumatic CE. PRM was able to detect $\mathrm{CE}$ when classical averaged approach failed. Our data showed that a single model of experimental TBI induced several types of CE evolutions. PRM could identify specific subgroups for clinical practice or for future experimental trials.

\section{Grant Acknowledgment}

'Fondation Gueules Cassées'. 


\section{Authors' details}

${ }^{1} \mathrm{CHU}$ Grenoble, Anesthesia-Intensive Care, Grenoble, France. ${ }^{2}$ University of Grenoble, Grenoble Institute of Neuroscience, Grenoble, France.

Published: 1 October 2015

\section{References}

1. Lemasson B, Galbán CJ, Boes JL, Li Y, Zhu Y, Heist KA, et al: DiffusionWeighted MRI as a Biomarker of Tumor Radiation Treatment Response Heterogeneity: A Comparative Study of Whole-Volume Histogram Analysis versus Voxel-Based Functional Diffusion Map Analysis. Transl Oncol 2013, 6(5):554-561.

2. Foda A, Marmarou A: A new model of diffuse brain injury in rats. Part II: Morphological characterization. J Neurosurg 1994, 80(2):301-313.

doi:10.1186/2197-425X-3-S1-A440

Cite this article as: Grèze et al:: Parametric response map (PRM) is a promising tool for the monitoring of post traumatic cerebral oedema. Intensive Care Medicine Experimental 2015 3(Suppl 1):A440.

\section{Submit your manuscript to a SpringerOpen ${ }^{\mathcal{O}}$ journal and benefit from:}

- Convenient online submission

- Rigorous peer review

- Immediate publication on acceptance

- Open access: articles freely available online

- High visibility within the field

- Retaining the copyright to your article

Submit your next manuscript at $\gg$ springeropen.com 